\title{
Trends in Antidiabetic Prescription Patterns in Japan From 2005 to 2011
}

\section{Impact of the Introduction of Dipeptidyl Peptidase-4 Inhibitors}

\author{
Takahide Kohro, ${ }^{1}$ MD, Tsutomu YamaZaKi, ${ }^{2}$ MD, Hiroki Sato,${ }^{3}$ MSc, Kenji Harada, ${ }^{4}$ MD, \\ Kazuhiko OHe, ${ }^{5} \mathrm{MD}$, Issei Komuro, ${ }^{6} \mathrm{MD}$, and Ryozo NAGAI, ${ }^{7} \mathrm{MD}$
}

\begin{abstract}
SUMMARY
There have been few reports concerning the trends in antidiabetic drug use in Japan. In 2009, a dipeptidyl peptidase-4 inhibitor (DPP4I), an antidiabetic with a new mechanism of action, was made available. This study was conducted to analyze the antidiabetic prescription trends in Japan in recent years and the influence of DPP4Is on those trends. We used monthly claims data obtained from a database company. Data from patients 20 years of age or older and who were prescribed antidiabetics were extracted and analyzed. A total of 18,457 patients were prescribed antidiabetics (mean age, 53.6 \pm 11.0 ). The sulfonylurea prescription rate decreased while that of biguanides increased. After the introduction of DPP4Is, use of these agents rapidly increased and the rate further increased one year after DPP4I introduction. DPP4Is also became the most prescribed antidiabetics for those prescribed antidiabetics for the first time. The decrease in the use of sulfonylureas and the increase in the use of biguanides are in accordance with trends observed in the United States and Europe, and probably reflect Japanese physicians' awareness of cumulating evidence gained from studies such as the UK Prospective Diabetes Study (UKPDS). The rapid increase in the DPP4I prescription rate might be the result of several factors including their safety profiles, which were highlighted in clinical studies published just prior to the drugs becoming available. However, there is little data regarding the efficacy of DPP4Is in reducing diabetes related complications, which should be determined in future studies. (Int Heart J 2013; 54: 93-97)
\end{abstract}

Key words: Clinical guidelines, Antidiabetics, Dipeptidyl peptidase-4 inhibitor, Prescription trends

$\mathrm{T}$

he goals of diabetes mellitus (DM) therapy are to prevent microvascular and macrovascular complications of hyperglycemia. Numerous clinical studies have been performed to compare the effectiveness of different drugs or to investigate the optimum level of blood glucose, and clinical guidelines have been built upon this evidence. However, there have been difficulties in the history of DM treatment. For example, sulfonylureas (SUs) are the oldest and most widely used oral antidiabetic agents because of their demonstrated blood glucose lowering effect, but there is some evidence that they can potentially damage pancreatic beta-cells, ${ }^{1-3)}$ which may cause secondary failure over time. ${ }^{4)}$ Several studies have shown that biguanides are also effective at lowering blood sugar and that they preserve beta-cell function. ${ }^{5-8)}$ It was also shown that metformin may be associated with fewer DM-related adverse events compared with other antidiabetics, ${ }^{9)}$ although it is still controversial. However, phenformin, which was the only biguanide available in the United States, was withdrawn from the market in the late 1970s because it was found to be associated with lactic acidosis with a high fatality rate. The withdrawal of phenformin negatively affected the use of biguanides in Japan. Biguanides were re-introduced into the US after a study demonstrated the safety and efficacy of metformin. ${ }^{10)}$ Thiazolidinediones (TZDs) were introduced in the 1990s, but each had its own problems. Troglitazone was withdrawn from the market because of the associated side effect of severe hepatitis. Recently, an association was suspected between rosiglitazone and increased myocardial infarction, ${ }^{11)}$ and while rosiglitazone has not been withdrawn from the market in the US, its use has been limited. In addition, pioglitazone was reported to be associated with an increased risk of bladder cancer. $^{12)}$

There have been several reports addressing the optimal blood sugar target level, and these reports have suggested that maintaining strict blood sugar control results in a smaller risk of microvascular complications. ${ }^{13,14)}$ However, no study has shown definitively that intensive lowering of blood glucose reduces the incidence of macrovascular complications such as

From the ${ }^{1}$ Department of Translational Research for Healthcare and Clinical Science, Graduate School of Medicine, The University of Tokyo, ${ }^{2}$ Clinical Research Support Center, The University of Tokyo Hospital, ${ }^{3}$ Department of Preventive Medicine and Public Health, National Defense Medical College, Departments of ${ }^{4}$ Health Care Safety Management, ${ }^{5}$ Medical Informatics and Economics, Division of Social Medicine, and ${ }^{6}$ Cardiovascular Medicine, Graduate School of Medicine, The University of Tokyo, Tokyo, and ${ }^{7}$ Jichi Medical University, Tochigi, Japan.

This work was supported by a grant from the Japanese Ministry of Economy, Trade and Industry and by the Japan Society for the Promotion of Science (JSPS), through the "Funding Program for World-Leading Innovative R\&D on Science and Technology (FIRST Program)", initiated by the Council for Science and Technology Policy (CSTP).

Address for correspondence: Takahide Kohro, MD, Department of Translational Research for Healthcare and Clinical Science, Graduate School of Medicine, The University of Tokyo, 7-3-1 Hongo, Bunkyo, Tokyo 113-8655, Japan.

Received for publication November 9, 2012.

Revised and accepted January 7, 2013. 
myocardial infarction or stroke. Three recent studies compared normal versus intensive blood glucose lowering, and these studies questioned the effectiveness of strict blood glucose control. $^{15-17)}$

In 2009, and for the first time in 10 years, antidiabetic agents with a new mechanism of action were introduced; these agents were dipeptidyl peptidase-4 inhibitors (DPP4Is) and glucagon-like peptide-1 (GLP-1) analogues.

Unlike the guidelines published by the American Diabetes Association (ADA) or the European Association for the Study of Diabetes (EASD), ${ }^{18,19)}$ the clinical guidelines published by the Japan Diabetes Society (JDS) do not explicitly state which oral agent should be used as first line therapy for newly diagnosed type 2 DM patients. Thus, we investigated the prescribing trends for antidiabetic agents in recent years in Japan, and the impact of DPP4Is on these trends. To perform this research, we analyzed information, collected in Japan from 2005 to 2011, in a large claims database.

\section{MethodS}

Data source and management: Monthly claims data (from January 2005 to October 2011) were obtained from a database company called Japan Medical Data Center (JMDC). ${ }^{20,21)}$ The population covered by the database consisted of insurees and their dependents belonging to one of several health insurance unions in Japan. The database was expanded in 2008, 2009 and 2010, and subjects from different health insurance unions were added. For each patient, the data consisted of an encrypted personal identifier, age and gender, a description of the procedures performed, diagnosis name and diagnosis code according to the World Health Organization's International Classification of Diseases (ICD10), and prescribed drugs. Drug information included the month and year of prescription, brand name and generic name, dosage, and number of days prescribed. Of the total one million patients in the database, information on those patients whose diagnosis name included 'diabetes mellitus' at least once and who were 20 years of age or older was initially extracted. However, not all patients with a DM diagnosis were prescribed antidiabetics, so the group was restricted to those who were prescribed any kind of antidiabetic agent for at least one month $(n=18,457)$. Most prescription periods ranged from 14 days to 3 months. We determined the prescription rates for each class of antidiabetic per patient per month. To do this, multiple prescriptions for a given drug during a single month were counted only once. For prescription periods of two months' duration, data were generated for the month following the first month and they were added to the original database. For prescription periods of 3 or more months' duration, data were generated for the two months following the initial month and they were added to the original database. Patients who were treated for DM for the first time were identified as those who were insured for more than 3 months without any antidiabetic prescriptions and then were prescribed at least one type of antidiabetic agent for at least 3 months. Data from patients who had undergone coronary revascularization were extracted by searching the procedure database using codes representing either a form of percutaneous coronary intervention or coronary artery bypass graft operation. Data from patients who were on maintenance dialysis were extracted by searching the procedure database using a code representing a special fee for maintenance dialysis. Data from patients who were prescribed at least one type of antihypertensive drug or any of the statin drugs were extracted by searching the prescription database.

Statistical analysis: Analysis of covariance (ANCOVA) was used to test the slope equality of DPP4I usage trends between clinics and academic hospitals.

The average of continuous variables is shown as the average \pm standard deviation. All $P$ values for statistical tests were two-sided. All statistical analyses were performed using Stata 12.1 (Stata Corporation, College Station, TX, USA).

Ethical considerations: The data used in this study were completely de-identified and informed consent from the subjects was not necessary. This study was approved by the ethics committee of the University of Tokyo.

\section{RESULTS}

Table I shows the patient background information. The mean age of the overall population was $53.6 \pm 11.0$ years, and the average age of females was slightly but significantly higher than that of males $(P=0.0001)$. There was a similar proportion of male $(0.8 \%)$ and female patients $(0.9 \%)$ who were on chronic hemodialysis $(P=0.417)$. The rate of coronary revascularization for males was nearly 3 times $(2.2 \%)$ that of females $(0.8 \%)$. A similar proportion of patients were prescribed at least one type of antihypertensive (51.0\% for males, $50.1 \%$ for females, $P=0.233$ ).

As shown in Figure 1, the use of SUs was declining and the use of biguanides was gradually increasing during the observation period. The use of alpha glucosidase inhibitors (GIs) abruptly decreased in January 2008, which is the result of adding a different population to the database. When addition of the new population was taken into account, the use of alpha-GIs remained almost constant until the end of 2009 and gradually

Table I. Patient Characteristics

\begin{tabular}{lcccc}
\hline & Total & Male & Female & $P^{\mathrm{a}}$ \\
\hline$n$ & 18457 & 11991 & 6466 & \\
Mean age & $53.6 \pm 11.0$ & $52.3 \pm 10.5$ & $53.2 \pm 12.8$ & $<0.0001^{\mathrm{b}}$ \\
Chronic hemodialysis & $152(0.8)$ & $94(0.8)$ & $58(0.9)$ & $0.417^{\mathrm{c}}$ \\
Coronary revascularization & $312(1.7)$ & $263(2.2)$ & $49(0.8)$ & $<0.0001^{\mathrm{c}}$ \\
Antihypertensives & $9356(50.7)$ & $6117(51.0)$ & $3239(50.1)$ & $0.233^{\mathrm{c}}$ \\
Statin & $7531(40.8)$ & $4612(38.5)$ & $2919(45.1)$ & $<0.0001^{\mathrm{c}}$ \\
\hline
\end{tabular}

Numbers in parentheses show the percentage of subjects in each group. a: test for the difference between male and female, b: tested by unpaired $t$-test, and c: tested by chi-square test. 


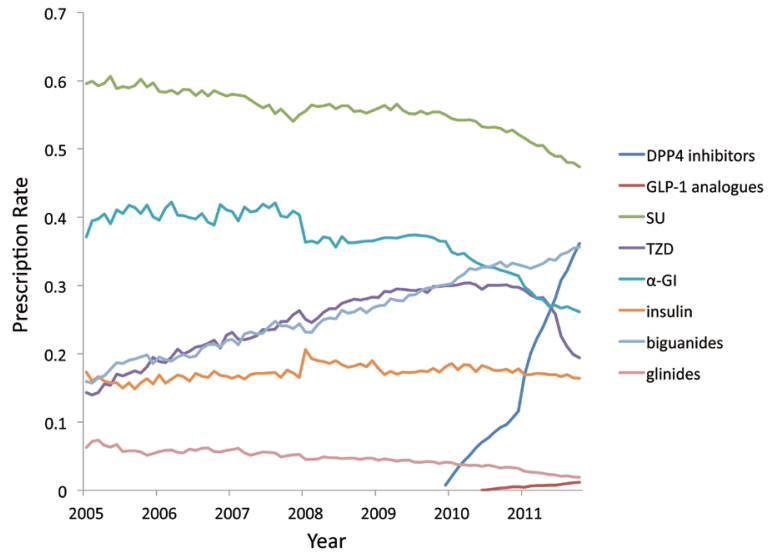

Figure 1. Prescription rates of each antidiabetic drug class. The abrupt change observed in January 2008 is due to the addition of a different population to the database. DPP4 indicates dipeptidyl peptidase-4; GLP-1, glucagon-like peptide-1; SU, sulfonylurea; TZD, thiazolidinedione; and $\alpha$-GI, alpha glucosidase inhibitor.
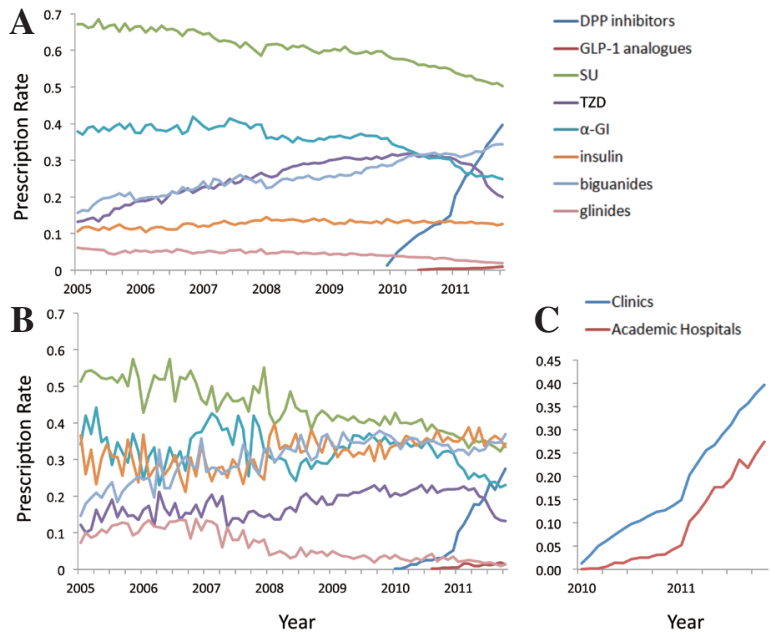

Figure 2. A: Clinic prescription rates for each antidiabetic class. Clinic in Japan are defined as medical institutions that do not possess any hospital beds or that possess up to 19 hospital beds. B: Academic hospital prescription rates for each antidiabetic class. C: Clinic and academic hospital prescription rate for dipeptidyl peptidase-4 inhibitors.

decreased thereafter. The use of TZDs increased until the end of 2009, stayed constant for approximately one year, and then started to decrease rapidly after the start of 2011. Glinide use had been slowly declining throughout the observation period. Insulin use remained relatively constant during the observation period.

We investigated the differences in the prescription pattern among different types of medical institutions. The claims database contained information on the type of medical institution for each prescription. Using this information, the prescription rate for each antidiabetic class was calculated. Figure 2A shows the rates for clinics and Figure $2 \mathrm{~B}$ shows those for academic hospitals. Clinics in Japan are defined as medical institutions that either do not possess any hospital beds or that possess up to 19 hospital beds. Several differences were observed. First, insulin use was much higher in academic hospitals than

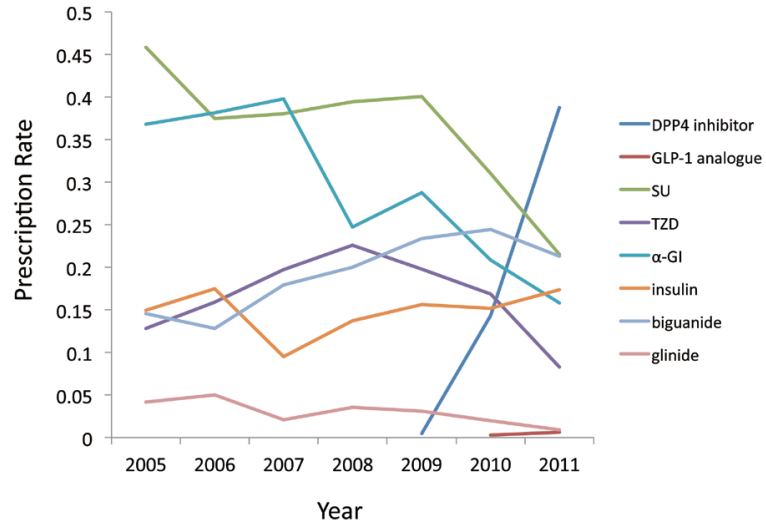

Figure 3. The prescription rate of each antidiabetic class used in the first month of diabetes mellitus treatment.

Table II. Distribution of the Number of Anti-Diabetics Which Were Prescribed in the First Month of Diabetes Mellitus Treatment

\begin{tabular}{cc}
\hline Number of drugs & $n(\%)$ \\
\hline 1 & $4550(79.64)$ \\
2 & $870(15.23)$ \\
3 & $239(4.18)$ \\
4 & $46(0.81)$ \\
5 & $8(0.14)$ \\
\hline
\end{tabular}

Numbers in parentheses show the percentage of subjects of the total number of patients who were initially treated for diabetes mellitus after diagnosis $(n=5713)$.

in clinics. Second, SU use was consistently lower in academic hospitals compared to clinics, but their use was decreasing in both groups. Third, the rate at which DDP4 inhibitor use was increasing was significantly different between the two groups (Figure 2C). When the slopes of the DPP4Is use from December 2009 to December 2010 were compared between clinics and academic hospitals, a significant difference was observed $(P<0.0001)$.

We then investigated the trend in the use of antidiabetics in the first month of DM treatment. Figure 3 shows that, in 2005, SUs were the most prescribed antidiabetics as a first line therapy, followed by alpha-GIs. However, the prescription rates of both drugs decreased during the observation period. DPP4Is, which became available at the end of 2009, rapidly became the most prescribed antidiabetic agents for initial treatment of DM patients. The number of agents prescribed in the first month of initial DM therapy included monotherapy for nearly $80 \%$ of patients, and prescription of 3 or more drugs for approximately $5 \%$ of patients (Table II).

\section{Discussion}

This is the first report on the trends in prescription of antidiabetic drugs from 2005 to 2011 in Japan. It reveals that the use of SUs is slowly declining and that of biguanides is gradually increasing, while the use of DPP4Is increased immediately after they became available and further increased after one year of availability. The first drug of choice has also changed and 
DPP4Is are now prescribed most often for initial treatment in DM patients. Differences in prescription patterns between different types of medical institutions were also observed.

The increase in the use of biguanides and the decrease in the use of SUs have also been reported in studies in the United States and Europe. ${ }^{22-24)}$ This is most likely because of the cumulating evidence gained from studies such as the UK Prospective Diabetes Study (UKPDS), ${ }^{9}$ as well as the consensus algorithm published by the ADA and EASD, which states that metformin should be used as the first drug in type $2 \mathrm{DM}$ patients. ${ }^{18,19)}$ However, unlike studies in the United States and Europe, our study in Japanese showed that SUs are still used more frequently than biguanides; this is probably because decreased insulin secretion capacity takes a definite role in the development of type 2 diabetes in Japan, while Caucasian type 2 DM patients suffer the disease primarily due to insulin resistance frequently associated with obesity. ${ }^{25}$

The dramatic increase in the use of DPP4Is after their availability may have been caused by several factors. Possibly one of the largest factors is the results of several clinical studies that were published just prior to the availability of DPP4Is, which compared intensive versus usual hyperglycemic control. ${ }^{15-17)}$ Unexpectedly, none of the studies showed that intensively lowering blood glucose levels reduced cardiovascular events. In the ACCORD study, it was shown that overall death was significantly increased in the intensive lowering group, which led to the early termination of the study. ${ }^{17)}$ The exact reasons why such results were observed remain unclear, but several explanations such as the higher frequency of hypoglycemia or weight gain in the intensive therapy group have been proposed. ${ }^{26)}$ DPP4Is theoretically do not induce hypoglycemia or weight gain when used alone, which might have led physicians to choose them preferentially over other antidiabetics after the publication of these studies. That reduced insulin secretion rather than insulin resistance plays a greater role in the pathophysiology in Japanese type 2 DM patients ${ }^{25}$ may also be one of the reasons for the rapid adoption of DPP4Is because DPP4Is are agents that stimulate the secretion of insulin from the pancreas.

The further increase in DPP4I use one year after they became available in Japan is likely a result of a Japanese law that, for one year, restricts the prescription of any new drug introduced into the market to 14 days per doctor's visit. It is usually much more difficult for larger hospitals, specialized hospitals or academic hospitals to follow their patients on a biweekly basis than for small clinics to do so, which likely resulted in the difference between the prescription increment rate between smaller clinics and academic hospitals (Figure 2C). That the increment rate was curbed for a year shows that the law is effective in reducing the number of prescriptions for that period, although if it was actually translated into more careful monitoring and fewer side effects remains unknown. Also, the discrepancy in increment rates between smaller clinics and academic hospitals, which have a higher rate of physicians with a specialty in DM management, raises some concerns on the law's effectiveness; specialists at the academic hospitals are more likely to be denied the opportunity to use new drugs in the early phase of its availability.

The use of TZDs was gradually increasing until the introduction of DPP4Is, and remained almost constant for the following year. However, after the restriction on the DPP4I pre- scription period was lifted, the use of TZDs began to decrease, and this trend was accelerated after the report of a TZD-associated increase in bladder cancer. ${ }^{12)}$ The use of alpha-GIs also remained constant until the introduction of DPP4Is (the decrease observed in January, 2008 was due to the addition of a different population to the database) and their use started to decrease after the introduction of DPP4I. TZDs and alpha-GIs are considered antidiabetics that do not induce hypoglycemia when used alone; this is a property that the DPP inhibitors also have. However, TZDs are known to be associated with increased heart failure, ${ }^{27)}$ weight gain ${ }^{28)}$ and bone fracture, ${ }^{29)}$ while there is no known association between DPP4Is and these adverse effects. Alpha-GIs must be taken 3 times a day, which may reduce the adherence, ${ }^{30,31)}$ while 3 of the 4 DPP4Is that are available in Japan can be taken once daily. Thus, it is possible that DPP4Is can be substituted for these antidiabetic drugs that do not cause hypoglycemia, but without the adverse effects associated with TZDs and with possibly better adherence rates than alpha-GIs.

In conclusion, although the Japanese guidelines do not clearly state what class of drug should be used for the treatment of DM patients, the use of biguanides has been steadily increasing, probably reflecting the physicians' awareness of cumulating evidence gained from studies such as UKPDS ${ }^{9)}$ The introduction of DPP4Is dramatically changed the prescription pattern for the treatment of DM patients, probably due to their favorable properties such as low probability of inducing hypoglycemia and weight gain, and infrequent dosing. However, whether these agents improve the outcomes of DM patients is still unknown, and future studies are required to determine their effect, especially when considering that the cost of DPP4Is is higher than that of other antidiabetic agents. ${ }^{32)}$

Study limitations: This study has several limitations. First, the database used in this study included only patients who were employed by large companies and it did not include patients employed in small businesses, the self-employed, or retirees. Thus, careful interpretation is required when extrapolating the results of this study to the whole population. Second, no information regarding the level of hyperglycemic control was available, and we could not investigate if the change in prescribing patterns resulted in better or worse glycemic control.

\section{REFERENCES}

1. Efanova IB, Zaitsev SV, Zhivotovsky B, et al. Glucose and tolbutamide induce apoptosis in pancreatic beta-cells. A process dependent on intracellular Ca2+ concentration. J Biol Chem 1998; 273: 33501-7.

2. Maedler K, Carr RD, Bosco D, Zuellig RA, Berney T, Donath MY. Sulfonylurea induced beta-cell apoptosis in cultured human islets. J Clin Endocrinol Metab 2005; 90: 501-6.

3. Del Guerra S, Marselli L, Lupi R, et al. Effects of prolonged in vitro exposure to sulphonylureas on the function and survival of human islets. J Diabetes Complications 2005; 19: 60-4.

4. Kahn SE, Haffner SM, Heise MA, et al. Glycemic durability of rosiglitazone, metformin, or glyburide monotherapy. N Engl J Med 2006; 355: 2427-43.

5. Marchetti P, Del Guerra S, Marselli L, et al. Pancreatic islets from type 2 diabetic patients have functional defects and increased apoptosis that are ameliorated by metformin. J Clin Endocrinol Metab 2004; 89: 5535-41.

6. Lupi R, Del Guerra S, Fierabracci V, et al. Lipotoxicity in human 
pancreatic islets and the protective effect of metformin. Diabetes 2002; 51 Suppl 1: S134-7.

7. Patanè G, Piro S, Rabuazzo AM, Anello M, Vigneri R, Purrello F Metformin restores insulin secretion altered by chronic exposure to free fatty acids or high glucose: a direct metformin effect on pancreatic beta-cells. Diabetes 2000; 49: 735-40.

8. Lupi R, Del Guerra S, Tellini C, et al. The biguanide compound metformin prevents desensitization of human pancreatic islets induced by high glucose. Eur J Pharmacol 1999; 364: 205-9.

9. Effect of intensive blood-glucose control with metformin on complications in overweight patients with type 2 diabetes (UKPDS 34). UK Prospective Diabetes Study (UKPDS) Group. Lancet 1998; 352: 854-65.

10. DeFronzo RA, Goodman AM. Efficacy of metformin in patients with non-insulin-dependent diabetes mellitus. The Multicenter Metformin Study Group. N Engl J Med 1995; 333: 541-9.

11. Nissen SE, Wolski K. Effect of rosiglitazone on the risk of myocardial infarction and death from cardiovascular causes. N Engl J Med 2007; 356: 2457-71.

12. Lewis JD, Ferrara A, Peng T, et al. Risk of bladder cancer among diabetic patients treated with pioglitazone: interim report of a longitudinal cohort study. Diabetes Care 2011; 34: 916-22.

13. Intensive blood-glucose control with sulphonylureas or insulin compared with conventional treatment and risk of complications in patients with type 2 diabetes (UKPDS 33). UK Prospective Diabetes Study (UKPDS) Group. Lancet 1998; 352: 837-53.

14. Ohkubo Y, Kishikawa H, Araki E, et al. Intensive insulin therapy prevents the progression of diabetic microvascular complications in Japanese patients with non-insulin-dependent diabetes mellitus: a randomized prospective 6-year study. Diabetes Res Clin Pract 1995; 28: 103-17.

15. Duckworth W, Abraira C, Moritz T, et al. Glucose control and vascular complications in veterans with type 2 diabetes. $\mathrm{N}$ Engl J Med 2009; 360: 129-39.

16. Patel A, MacMahon S, Chalmers J, et al. Intensive blood glucose control and vascular outcomes in patients with type 2 diabetes. N Engl J Med 2008; 358: 2560-72.

17. Gerstein HC, Miller ME, Byington RP, et al. Effects of intensive glucose lowering in type 2 diabetes. N Engl J Med 2008; 358 : 2545-59.

18. Nathan DM, Buse JB, Davidson MB, et al. Management of hyperglycemia in type 2 diabetes: A consensus algorithm for the initiation and adjustment of therapy: a consensus statement from the American Diabetes Association and the European Association for the Study of Diabetes. Diabetes Care 2006; 29: 1963-72.

19. Nathan DM, Buse JB, Davidson MB, et al. Medical management of hyperglycemia in type 2 diabetes: a consensus algorithm for the initiation and adjustment of therapy: a consensus statement of the American Diabetes Association and the European Association for the Study of Diabetes. Diabetes Care 2009; 32: 193-203.

20. Kimura S, Sato T, Ikeda S, Noda M, Nakayama T. Development of a database of health insurance claims: standardization of disease classifications and anonymous record linkage. J Epidemiol 2010; 20: 413-9.

21. Japan Medical Data Center. Available at: http://www.jmdc.co.jp/ Accessed December 18, 2012.

22. Baviera M, Monesi L, Marzona I, et al. Trends in drug prescriptions to diabetic patients from 2000 to 2008 in Italy's Lombardy Region: a large population-based study. Diabetes Res Clin Pract 2011; 93: 123-30.

23. Filion KB, Joseph L, Boivin JF, Suissa S, Brophy JM. Trends in the prescription of anti-diabetic medications in the United Kingdom: a population-based analysis. Pharmacoepidemiol Drug Saf 2009; 18: 973-6.

24. Alexander GC, Sehgal NL, Moloney RM, Stafford RS. National trends in treatment of type 2 diabetes mellitus, 1994-2007. Arch Intern Med 2008; 168: 2088-94.

25. Fukushima M, Suzuki H, Seino Y. Insulin secretion capacity in the development from normal glucose tolerance to type 2 diabetes. Diabetes Res Clin Pract 2004; 66 Suppl 1: S37-43. (Review)

26. Hoogwerf BJ. Does intensive therapy of type 2 diabetes help or harm? Seeking accord on ACCORD. Cleve Clin J Med 2008; 75 : 729-37. (Review)

27. Hernandez AV, Usmani A, Rajamanickam A, Moheet A. Thiazolidinediones and risk of heart failure in patients with or at high risk of type 2 diabetes mellitus: a meta-analysis and meta-regression analysis of placebo-controlled randomized clinical trials. Am J Cardiovasc Drugs 2011; 11: 115-28. (Review)

28. Mitri J, Hamdy O. Diabetes medications and body weight. Expert Opin Drug Saf 2009; 8: 573-84. (Review)

29. Bodmer M, Meier C, Kraenzlin ME, Meier CR. Risk of fractures with glitazones: a critical review of the evidence to date. Drug Saf 2009; 32: 539-47. (Review)

30. Donnan PT, MacDonald TM, Morris AD. Adherence to prescribed oral hypoglycaemic medication in a population of patients with Type 2 diabetes: a retrospective cohort study. Diabet Med 2002; 19: 279-84.

31. Dezii CM, Kawabata H, Tran M. Effects of once-daily and twicedaily dosing on adherence with prescribed glipizide oral therapy for type 2 diabetes. South Med J 2002; 95: 68-71.

32. Karagiannis T, Paschos P, Paletas K, Matthews DR, Tsapas A. Dipeptidyl peptidase-4 inhibitors for treatment of type 2 diabetes mellitus in the clinical setting: systematic review and meta-analysis. BMJ 2012; 344: e1369. 